\title{
P16 Positive by Immunohistochemistry
}

National Cancer Institute

\section{Source}

National Cancer Institute. p16 Positive by Immunohistochemistry. NCI Thesaurus. Code C158943.

An indication that CDKN2A-p16 expression has been detected in a sample using immunohistochemical techniques. 